\title{
Comparative Evaluation of Chlorhexidine and its Combination with Chitosan as Intracanal Medicaments on Enterococcus faecalis in Endodontic Retreatment
}

\author{
Kapadia M, Srirekha A*, Lekha S, Savitha B, Vijay R and Archana S \\ Department of Conservative Dentistry and Endodontics, The Oxford Dental College, Bomanhalli, Bangalore- 560068, India
}

\begin{abstract}
Aim: The aim of this study was to evaluate the efficacy and synergistic effect of chitosan in combination with chlorhexidine gel and chlorhexidine alone as intra canal medicaments in retreatment cases.

Materials \& Methods: Thirty single rooted teeth indicated for root canal retreatment were selected. After gutta percha (GP) removal, a pre-treatment sample (S1) was taken. Following which cleaning and shaping using sodium hypochlorite and EDTA was done and post-instrumentation sample (S2) was collected. The teeth were randomly divided into two groups Group I $(n=15): 2 \%$ chlorhexidine gluconate gel Group II $(n=15): 2 \%$ chlorhexidine gluconate gel and $2 \%$ chitosan (1:1). Post medication sample (S3) was taken after seven days and sent for quantitative PCR evaluation for $E$. faecalis. The data was statistically analyzed using Mann Whitney $U$ test and Kruskal Wallis test $(p<0.05)$.
\end{abstract}

Results: The results from Kruskal Wallis test indicate comparison of bacterial counts in group 1 and group2 for $S 1$, $\mathrm{S} 2$ and S3 showed bacterial reduction count from S1 to S2. The bacterial count difference between S1- S3 was better in group 2 compared to group 1. However, it was not statistically significant $(p<0.213)$.

Conclusion: Cleaning and shaping and irrigation along with use of intracanal medicament resulted in decrease in the mean $E$. faecalis in both the groups. $2 \%$ chlorhexidine gluconate gel in combination with chitosan performed better than chlorhexidine alone against $E$. faecalis, However, it is not statistically significant. $(p<0.213)$.

Keywords: Retreatment; Polymerase chain reaction; ProTaper retreatment files Chlorhexidine gluconate; Chitosan

\section{Introduction}

Bacteria remaining within the root canal system are a significant factor in endodontic failures. Hence, retreatment of endodontically treated teeth is a major challenge to clinicians. Enterococcus faecalis has been most frequently identified in $12 \%$ to $77 \%$ of the cases in canals of root-filled teeth with periapical lesions either by culture or molecular methods [1]. Molecular analyses have revealed the presence of uncultivable or difficult-to-grow bacteria in infected root canals, providing the opportunity to obtain a significant amount of new information on endodontic microbiota [2]. Recent advances in cellular and molecular biological methods revealed real- time quantitative Polymerase Chain Reaction (PCR) is 10-100 times more sensitive [3]. Cleaning and shaping effectively reduce microbiota but these procedures do not completely eliminate bacteria in the lateral and accessory root canals, isthmi, and apical deltas.

Intracanal medicaments are used as an antibacterial agent to eliminate residual bacteria in a root canal after instrumentation and irrigation, to render any remaining canal content inert [4]. Chlorhexidine $(\mathrm{CHX})$ is a potent antimicrobial frequently used in endodontics [5]. It is a broad spectrum antibacterial agent that is effective against both E. faecalis and Candida albicans. In addition to its immediate action on bacteria, chlorhexidine can be adsorbed onto and subsequently released from dental tissues, resulting in substantive antibacterial activity or "substantivity" [6]. Chitosan is an amino polysaccharide biopolymer, which displays excellent biocompatibility, physical stability and processability. It has widely been used in the area of dentistry as a bioadhesive; viscosity-enhancer; for prolonged drug release in buccal cavity; permeabilizer; antimicrobial; anti-adhesive; anti-cariogenic; for treatment of periodontal diseases/oral candidiasis/ tooth mobility and reduction of plaque formation [7]. However its role in endodontics in vivo has not been subjected to adequate scrutiny. The in vivo evaluation of synergistic effect of chitosan and its combination with $\mathrm{CHX}$ as intracanal medicament against the resistant bacteria like E,faecalis will have important clinical implications in nonsurgical endodontiv retreatment.

Hence, the aim of this clinical study is to evaluate the antimicrobial efficacy of chlorhexidine and its combination with chitosan as intracanal medicament in response to E. faecalis in retreatment cases.

\section{Materials and Methods}

Thirty patients between 19-65 years of age requiring root canal retreatment were selected for the study. All selected teeth were single rooted which were symptomatic or asymptomatic and had history of root canal treatment and showed periapical radiolucency upto $4 \mathrm{~mm}$. Ethical clearance was obtained from the institutional ethical committee. All patients were explained about the treatment procedure and informed consent was taken.

The selected tooth was isolated with a rubber dam. The crown and surrounding rubber dam were disinfected with $30 \%$ hydrogen peroxide for 30 s followed by vigorous swabbing of $3 \%$ sodium hypochlorite solution. Subsequently $5 \%$ sodium thiosulfate was used to inactivate the disinfectant. Access preparation was done with a sterile Endo access

*Corresponding author: Dr Srirekha A, M.D.S, Professor and Head Department of Conservative Dentistry and Endodontics, The Oxford Dental College, Bomanhalli, Bangalore- 560068, India, Tel: 09620958898; E-mail: drsrirekha.a@gmail.com

Received July 16, 2018; Accepted July 26, 2018; Published July 28, 2018

Citation: Kapadia M, Srirekha A, Lekha S, Savitha B, Vijay R, et al. (2018) Comparative Evaluation of Chlorhexidine and its Combination with Chitosan as Intracanal Medicaments on Enterococcus faecalis in Endodontic Retreatment. Dentistry 8: 502. doi:10.4172/2161-1122.1000502

Copyright: (c) 2018 Kapadia M, et al. This is an open-access article distributed under the terms of the Creative Commons Attribution License, which permits unrestricted use, distribution, and reproduction in any medium, provided the original author and source are credited. 
Citation: Kapadia M, Srirekha A, Lekha S, Savitha B, Vijay R, et al. (2018) Comparative Evaluation of Chlorhexidine and its Combination with Chitosan as Intracanal Medicaments on Enterococcus faecalis in Endodontic Retreatment. Dentistry 8: 502. doi:10.4172/2161-1122.1000502

Page 2 of 4

bur (Dentsply Maillefer, Ballaigues, Switzerland) until root canal filling was exposed. On exposing the previous root filling, the second stage of disinfection was done for the operative field by vigorous swabbing of $3 \%$ sodium hypochlorite solution. Subsequently $5 \%$ sodium thiosulfate was used as a neutralizer.

Gutta percha (GP) was removed with Protaper Retreatment files in sequence of $\mathrm{D}_{1}, \mathrm{D}_{2}$ and $\mathrm{D}_{3}$ (Protaper universal, Dentsply, India) as per the manufacturer's instructions and no chemical solvents were used in retrival of gutta percha. A periapical radiograph was taken to check the complete removal of GP. First Sample (S1) contained retrived GP, dentinal shavings and paper point which were transferred aseptically to tubes containing transport media. Working length was measured by preoperative radiographs and \# 15 file was inserted into the root canal $0.5 \mathrm{~mm}$ short of the root apex and working length was confirmed using electronic apex locator (Root ZX mini, J Morita Corp, Tokyo, Japan).

Canals were irrigated using $5 \mathrm{ml} \mathrm{3 \%}$ sodium hypochlorite and 17\% EDTA during instrumentation. To inactivate sodium hypochlorite, 3 $\mathrm{ml}, 5 \%$ sodium thiosulphate was used followed by flushing with saline. After drying the root canal with sterile paper points a second root canal sample (S2) was taken. Patients were randomly divided into two study groups:-

\section{Group I ( $n=15): 2 \%$ chlorhexidine gluconate gel}

Group II ( $n=15): 2 \%$ chlorhexidine gluconate gel and 2\% chitosan (Everest Biotec, Bangalore) (1:1)

Intracanal medicaments in Group I was placed in gel form and in Group II in paste form. The access was then sealed with sterile cotton pellet and Resin modified Glass Ionomer Cement.

Patients were recalled after 7 days and all restorations were checked for integrity and dislodged restorations were excluded from the study. Restoration was removed using sterile bur and residual intra canal medicament was flushed out with saline and the canal walls were lightly filed with no. $30 \mathrm{H}$ file.

In Group I: 2 ml of $3 \%$ Tween 80 was used to neutralize chlorhexidine gluconate, over a period of 5 minutes followed by irrigation with sterile saline. In Group II: along with $2 \mathrm{ml}$ of $3 \%$ Tween 80 , serial dilution using saline was used to neutralize the combination.

To evaluate residual burden of Enterococcus faecalis microorganism post medicament sample (S3) was taken. After sample collection root canal treatment was completed. None of the patients in this study needed intervention during treatment due to flare up.

Quantitative Real time Polymerase Chain Reaction

Total genomic DNA from the bacteria was isolated by N- Cetyl- N, $\mathrm{N}, \mathrm{N}$-trimethyl- ammonium bromide (CTAB) method. The quantity of the isolated DNA was checked in UV-VIS spectrophotomer (Vivaspec Biophotometer, Germany). From the stock $1 \mu \mathrm{l}$ DNA was mixed with $49 \mu \mathrm{l}$ sterile distilled water to get 50 times dilution. The A260/A280 ratio was recorded to check the purity of DNA preparation.

Real-time PCR was performed using a StepOne, Applied Biosystems USA. Real-time PCR was carried out with an initial incubation of 10 min at $95^{\circ} \mathrm{C}$ followed by 35 cycles consisting of denaturing at $95^{\circ} \mathrm{C}$ for 10 seconds; annealing at $60^{\circ} \mathrm{C}$ for $5 \mathrm{sec}$ followed by amplification at $72^{\circ} \mathrm{C}$ for 30 seconds. Amplification and detection were carried out in optical-grade 48 well plates in an ABI Prism Sequence Detection System. After amplification, a melting curve analysis was performed to determine the specificity of the PCR products. Threshold cycle (Ct) analysis of all samples were either set at 0.5 relative fluorescence units or left to automatic detection by the system. Quantification analysis was performed using StepOne Plus software.

\section{Results}

Statistical software IBM SPSS statistics 20.0 (IBM Corporation, Armonk, NY, USA) was used for the analyses of the data and Microsoft word and Excel were used to generate graphs, tables etc.

Results in Table 1 indicate that the percentage reduction of bacteria in samples dropped from S1 to S2. Decrease in microbial load was seen from S2-S3 at the end of 7 days in both groups. The comparison of bacterial counts (Mean rank) in group 1 and group 2 at different time interval using Kruskal Wallis test is given in Graph 1 and 2

The results show that the bacterial count difference of group 1 and group 2 is 13.50 and 17.50 respectively (Table 2). However, it is not statistically significant $(\mathrm{p}<0.05)$.

\section{Discussion}

Endodontic failures can be attributable to inadequacies in shaping, cleaning and obturation, and also reinfection of the root canal system when the coronal seal is lost after completion of root canal treatment [8]. To increase the efficiency of instrumentation, root canal irrigating solutions and intracanal medicaments are used to eliminate the bacteria from the root canals [9]. This can be ascribed to the usual inability of instruments and irrigants in cleaning and disinfecting anatomical variables, which are very common in the apical portion of the root canals $[10,11]$.

In the present study the results showed a reduction in microbial load in both groups. In Group 1 mean rank for S1, S2 was 25.07, 23.07 (Graph 1) and percentage reduction of bacterial load between S1-S2 was $38.40 \%$, (Table 1) while group 2 showed the mean rank for S1, S2 was 26.33, 23.03 (Graph 2) and the percentage reduction of bacterial load between S1-S2 being $43.15 \%$ respectively (Table 1 ). Various clinical studies that have reported that chemomechanical procedures reduce microorganisms in the root canal system $[12,13]$ Siqueira et al. reported that $4 \% \mathrm{NaOCl}$ was significantly more effective than saline solution in disinfecting root canals inoculated with $E$. faecalis [14]. Shuping et al. showed after instrumentation with rotary $\mathrm{NiTi}$ instruments along with $\mathrm{NaOCl}$ irrigation, $61.9 \%$ of canals were

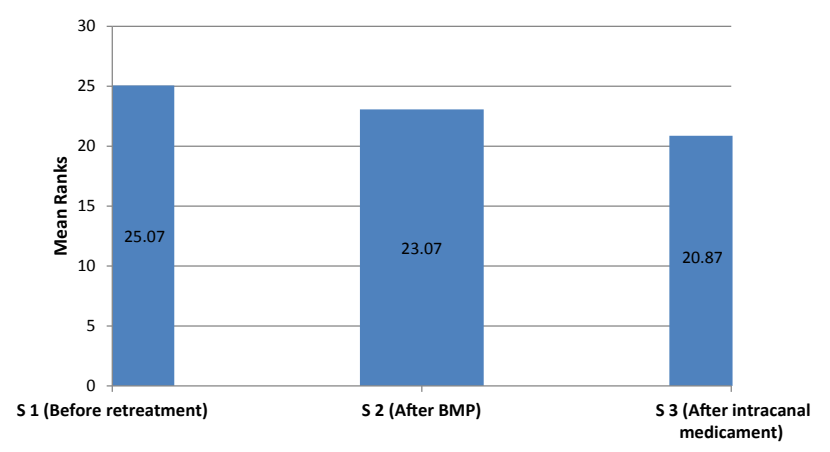

Group 1: Chlorhexidine.

Graph 1: Comparison of bacterial counts (Mean rank) in group 1 at different time interval using Kruskal Wallis test.

\begin{tabular}{|c|c|c|c|}
\hline Number of Groups & S1-S2 & S2-S3 & S1-S3 \\
\hline Group 1 & $38.40 \%$ & $32.54 \%$ & $70.94 \%$ \\
\hline Group 2 & $43.15 \%$ & $12.27 \%$ & $55.42 \%$ \\
\hline
\end{tabular}

Table 1: Percentage reduction in group 1 (chlorhexidine) and Group 2 (chlorhexidine and chitosan). 
Citation: Kapadia M, Srirekha A, Lekha S, Savitha B, Vijay R, et al. (2018) Comparative Evaluation of Chlorhexidine and its Combination with Chitosan as Intracanal Medicaments on Enterococcus faecalis in Endodontic Retreatment. Dentistry 8: 502. doi:10.4172/2161-1122.1000502

rendered bacteria-free [15]. Although the protocol used in this study reduced $E$. faecalis levels, the bacterium was not totally eliminated from the canal. This is because in spite of the thorough instrumentation tissue remnants can be localized in isthmuses, irregularities, dentinal tubules and lateral canals, which very often remain unaffected by instruments and irrigants [16]. In Group 1 mean rank for S3 was 20.87 (Graph 1) and percentage reduction of bacterial load between S2-S3 and S1-S3 is $32.54 \%$ and $70.94 \%$ respectively (Table 1 ). Similar results were found in a clinical study which showed $78 \%$ negative cultures after 7 days [17] Chlorhexidine has a broad spectrum antimicrobial effect targeting both gram positive and gram negative microorganisms [18]. It has marked effect against resistant microorganisms in the root canal such as E. faecalis, anaerobic bacteria and Candida albicans [19]. A study inferred that 2\% CHX gel was able to clean the root canal walls and their anatomic complexities effectively because of the viscosity of the gel, which promoted a better mechanical cleansing of the root canal, better removal of dentin debris and the remaining tissue. In addition, 2\% CHX gel has good antimicrobial property which can potentially disinfect the dentinal tubules and anatomical complexities during instrumentation as compared to solution form [20].

The results for Group 2 showed that the mean rank for S3 is 19.63 (Graph 2) and the percentage reduction of bacterial load between S2-S3 and S1-S3 is $12.27 \%$ and $55.42 \%$ respectively (Table 1 ). Combination of irrigants or medicaments decreases the development of resistant bacterial strains and produces synergistic effect, leading to long lasting antimicrobial action and sustained release of medicaments [21]. The spectrum of antimicrobial activity of chitosan and its derivatives extends to include filamentous fungi, yeasts and bacteria, being more active against gram-positive than gram-negative bacteria [22]. Combination of two medications may produce additive or synergistic effect whose antimicrobial action may last longer and also sustain the release of medicaments [23]. On intergroup comparison, the bacterial count difference between S1 and S3 of group 1 and group 2 is 13.50 and 17.50 respectively (Table 2). This result showed that Group

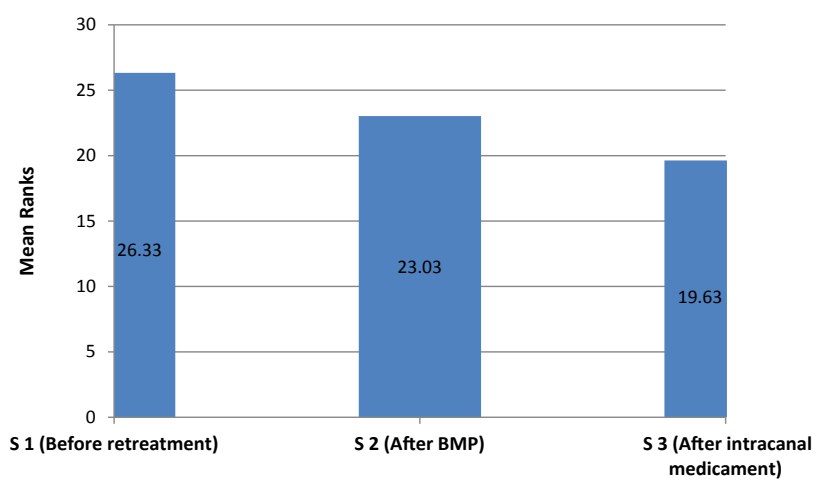

Group 2: Chlorhexidine and chitosan.

Graph 2: Comparison of bacterial counts (Mean rank) in group 2 at different time interval using Kruskal Wallis test.

\begin{tabular}{|c|c|c|}
\hline Groups & No. of samples & Mean rank \\
\hline Group 1: Chlorhexidine & 15 & 13.50 \\
\hline Group 2: Chlorhexidine + chitosan & 15 & 17.50 \\
\hline Z value & - & 1.244 \\
\hline P value & - & 0.213 \\
\hline
\end{tabular}

Table 2: Comparison of bacterial counts difference (Mean ranks) \{before retreatment (S1)- after placing intracanal medicament (S3)\} among both the groups using Mann Whitney $U$ test. $Z$ value (Standard deviation) P value (Statistical significance).
2 performed better than group 1 , however there is no statistically significant $(\mathrm{p}<0.213)$ decrease in microbial count.

An in vitro study was conducted to investigate the antimicrobial effectiveness of $2 \%$ CHX gel, $2 \%$ chitosan gel and their combination against Candida albicans and E. faecalis. It was established that release of chlorhexidine with chitosan was better than plain chitosan alone, combination of the two showed maximum inhibitory zone for Candida albicans and E. faecalis. This study advocated that $2 \%$ chlorhexidine gel in combination with $2 \%$ chitosan had better antimicrobial efficacy than either of them alone [23].

\section{Conclusion}

Within the limitations of this in vivo study the following conclusion can be drawn: Cleaning and shaping (S1-S2) and irrigation along with use of intracanal medicaments like CHX and its combination with chitosan resulted in reduced mean E. faecalis load in both the groups, however it was not statistically significant. Intergroup comparison showed 2\% CHX in combination with chitosan performed better compared to $\mathrm{CHX}$ as an intracanal medicament when used against $E$. faecalis, however it was not statistically significant $(\mathrm{p}<0.213)$.

\section{References}

1. Gomes BP, Pinheiro ET, Jacinto RC, Zaia AA, Ferraz CC, et al. (2008) Microbia analysis of canals of root-filled teeth with periapical lesions using polymerase chain reaction. $\mathrm{J}$ Endod 34: 537-540.

2. Kim SY, Shin Y, Lee CY, Jung IY (2013) In-vivo quantitative evaluation of live and dead bacteria in root canal infection by using propidium monoazide with real-time PCR. J Endod 39: 1359-1363.

3. Siqueria JF, Rôcas IN (2003) PCR methodology as a valuable tool for identification of endodontic pathogens. J Dent 31: 333-339.

4. Chong BS, Pittford TR (1992) The role of intracanal medication in root canal treatment. Int Endod J 25: 97-106.

5. Mohammadi Z, Abbott PV (2009) The properties and applications of chlorhexidine in endodontics. Int Endod J 42: 288-302.

6. Paquette L, Legner M, Fillery ED, Friedman S (2007) Antibacterial efficacy of chlorhexidine gluconate intracanal medication in vivo. J Endod 33: 788-795.

7. Raafat D, Sahl HG (2009) Chitosan and its antimicrobial potential- a critical literature survey. Microb Biotechnol 2: 186-201.

8. Alves J, Walton R, Drake D (1998) Coronal leakage: endotoxin penetration from mixed bacterial communities through obturated, post-prepared root canals. J Endod 24: 587-591.

9. Byström A, Sundqvist $G$ (1985) The antibacterial action of sodium hypochlorite and EDTA in 60 cases of endodontic therapy. Int Endod J 18: 35-40.

10. Ida RD, Gutmann JL (1995) Importance of anatomic variables in endodontic treatment outcomes: case report. Dent Traumatol 11: 199-203.

11. Siqueira JF, Araújo MC, Garcia PF, Fraga RC, Dantas CJ (1997) Histological evaluation of the effectiveness of five instrumentation techniques for cleaning the apical third of root canals. J Endod 23: 499-502.

12. Byström A, Sundqvist G (1981) Bacteriologic evaluation of the efficacy of mechanical root canal instrumentation in endodontic therapy. Scand J Dent Res 89: 321-328.

13. Peter LB, Wesselink PR (2002) Periapical healing of endodontically treated teeth in one and two visits obturated in the presence or absence of detectable microorganisms. Int Endod J 35: 660-667.

14. Siqueira JF, Machado AG, Silveira RM, Lopes HP, de Uzeda M (1997) Evaluation of the effectiveness of sodium hypochlorite used with three irrigation methods in the elimination of Enterococcus faecalis from the root canal, in vitro. Int Endod J 30: 279-282.

15. Shuping G, Ørstavik D, Sigurdsson A, Trope M (2000) Reduction of intracanal bacteria using nickel-titanium rotary instrumentation and various medications. J Endod 26: 751-755. 
Citation: Kapadia M, Srirekha A, Lekha S, Savitha B, Vijay R, et al. (2018) Comparative Evaluation of Chlorhexidine and its Combination with Chitosan as Intracanal Medicaments on Enterococcus faecalis in Endodontic Retreatment. Dentistry 8: 502. doi:10.4172/2161-1122.1000502

Page 4 of 4

16. Shaik J (2014) Comparative evaluation of antimicrobial efficacy of triple antibiotic paste and calcium hydroxide using chitosan as carrier against Candida albicans and Enterococcus faecalis: An invitro study. J Cons Dent 17: $335-339$

17. Barbosa CA, Goncalves RB, Siqueira JF Jr, De Uzeda M (1997) Evaluation of the antibacterial activities of calcium hydroxide, chlorhexidine, and camphorated paramonochlorophenol as intracanal medicament: A clinical and laboratory study. J Endod 23: 297-300.

18. Rôças IN, Siqueria JF (2011) Comparison of the in vivo antimicrobial effectiveness of Sodium Hypochlorite and Chlorhexidine used as root canal irrigants: a molecular microbiology study. J Endod 37: 143-150.

19. Singh RD, Khatter R, Bal RK, Bal CS (2013) Intracanal medications versus placebo in reducing postoperative endodontic pain- a double -blind randomized clinical trial. Braz Dent J 24: 25-29.
20. Ferraz CC, Figueiredo de Almeida Gomes BP, Zaia AA, Teixeira FB, de Souza-Filho FJ (2001) In vitro assessment of the antimicrobial action and the mechanical ability of chlorhexidine gel as an endodontic irrigant. J Endod 27: $452-455$.

21. Goodson J (1989) Pharmacokinetic principles controlling efficacy of oral therapy. J Dent Res 68: 1625-1632.

22. Lee DY, Spångberg LS, Bok YB, Lee CY, Kum KY (2005) The sustaining effect of three polymers on the release of chlorhexidine from a controlled release drug device for root canal disinfection. Oral Surg Oral Med Oral Pathol Oral Radiol Endod 100: 105-111.

23. Ballal NV, Kundabala M, Bhat KS, Acharya S, Ballal M, et al. (2009) Susceptibility of Candida albicans and Enterococcus faecalis to chitosan, chlorhexidine gluconate and their combination in vitro. Aus Endod J 35: 29-33. 